\title{
Towards Interoperability in Mobile Coupons: Enabling Cross Retailer Coupon Validation
}

\section{Conference Paper}

\section{Author(s):}

Fuchs, Klaus L.; Vuckovac, Denis; llic, Alexander

Publication date:

2017-10

Permanent link:

https://doi.org/10.3929/ethz-b-000198574

Rights / license:

In Copyright - Non-Commercial Use Permitted

Originally published in:

https://doi.org/10.1109/ICTC.2017.8190997 


\section{Towards Interoperability in Mobile Coupons: Enabling Cross Retailer Coupon Validation}

\author{
Fuchs, Klaus \\ Chair of Information Management \\ ETH Zurich \\ Zurich, Switzerland \\ fuchsk@ethz.ch
}

\author{
Vuckovac, Denis \\ Chair of Information Management \\ ETH Zurich \\ Zurich, Switzerland \\ dvuckovac@ethz.ch
}

\author{
Ilic, Alexander \\ Chair of Information Management \\ ETH Zurich \\ Zurich, Switzerland \\ ailic@ethz.ch
}

\begin{abstract}
A clear majority of coupons distributed by retailers remains exclusively redeemable at the retailer's own store network. Although interoperability among multiple retailers would promise added value for consumers, brands and retailers, technical gaps - primarily involving a lack of common standards and technical interfaces - render such interoperability unfeasible. However, shifting market forces have led to increased usage of mobile apps and retailers applying omni-channel strategies. In turn, leading to new proposed technical coupon standards that enable interoperable cross-retailer coupon distribution and validation. Interestingly, such interfaces have hardly been implemented or tested in a real-world, cross-retailer environment. Moreover, they have received little discussion in public discussions and related literature. This paper addresses and contributes to this gap by presenting findings from an interoperable couponing trial connecting 17 retailers, 8 different mobile applications and one brand, and discusses the chosen interface design necessary for implementation and coupon validation. Further practical pilots are planned to improve the specification and measure consumer and industry acceptance of coupon interoperability.
\end{abstract}

Keywords - interoperability, couponing, system architecture, interface standardization.

\section{MOTIVATION}

The main motivation of this paper is to extend the contemporary, normative definitions of retail coupons [1], [2] and recently conceptualized but not yet implemented crossretailer interface standards [3], [5], by presenting potential interface designs that enable cross-retailer coupon validation. To the best of the authors' knowledge, the implemented study conducted in Birmingham, UK, is the first publicly described technical implementation of an interoperable cross-retailer coupon ecosystem suggested in [3].

As of today, the coupon landscape remains a fragmented ecosystem consisting of isolated, disjunctive retailer/mobileapp partnerships [2]-[5], thereby technically preventing crossorganizational information exchange, such as coupon validation. In this work, relevant learnings from Internet-ofThings (IoT) [6], [7] implementations of similarly distributed and non-integrated IoT ecosystems are leveraged in order to enable a standardized information exchange between the different couponing stakeholders (e.g. brands, mobile apps, retailers, validators). Smart home automation [8]-[10] for example illustrates one such ecosystem, wherein information exchanges occur between devices and services that are provided by different parties [11], [12], and are usually solved through the provision of standardized APIs. The advantages of such cross-organizational information exchange are manifold: Automatic distribution and validation of coupons [3] decreases implementation effort of new coupon campaigns. It lowers operational costs by harmonizing coupon distribution [13]. Moreover, the standardization validation processes at the point of sale can prevent coupon fraud [14], [13] and administrative effort [13]. Furthermore, consumers benefit from enabling coupon interoperability making coupon redemptions at multiple outlets more convenient, thereby increasing redemption rates [15], [16].

\section{RELATED WORK}

The distribution of paper-based [19] or digital coupons [21] to incentivize consumer purchase [2] is a widely applied practice in the retail industry [17], [18]. Currently, over 320bn coupons are distributed annually in the US alone (2015) [19], [20], totaling in billion US-dollars worth of retail revenue and savings for consumers [2], [21], [22]. Despite distribution growth, consumers redeem coupons less frequently, if at all [13], [20], [23]. More than $90 \%$ of coupons remain unredeemed [20], [24]. Key reasons include a perceived lack of personalization [25] or low value of coupons [20], [22], [26], [27], redemption effort, decreased readership of printed media [20], and low availability of digital coupons [20].

However, digital coupons [22], [28], [29] have been found to promise superiority over their paper-based alternatives in regards to coupon personalization [2], [30], administrative costs, fraud prevention [2], distribution costs [22], [30] as well as ease-of-use [31], [32]. Despite the former reasons and the strong double-digit growth of mobile commerce [33], still only $20 \%-35 \%$ of consumers make use of digital coupons [20], [34], albeit $90 \%$ of consumers are willing to do so [35] and $80-90 \%$ 
own digital devices [36]. These circumstances have been explained by the reluctance of retailers to offer digital coupons, in part due to lacking technical capabilities for (third party) validation - both in-store or web-based [2], [14], lack of standards in the technical definition of a coupon object [2] and a lack of technical interfaces between brands, retailers and internet application providers [2], [37].

Until today, most contemporary coupon ecosystems are proprietary and local solutions that do not comply with existing and established retail standards [1], [2], [5] and therefore their adoption lacks acceptance. In contrast to other established retail standards (e.g. GTIN [38]), similar standardization of coupons have yet failed to be adopted majorly [1]-[3]. For example, Coupons.com [39] and Amazon [40] filed patents for a proprietary coupon management system handling. These did not include generating coupons at the retailer side nor printing paper coupons [2]. Another system [41] for the distribution of digital coupons based on the consumer's geographical position neglects the opportunity of remote-ordering (e.g. e-commerce). Apple's solution for storage, management and redemption of digital coupons through a mobile device [42], also neglects the still predominantly used paper-based coupons. [43] suggested to incorporate all coupon details, such as the expiration date, redemption locations, etc. into the coupon object, thereby neglecting usage of established standards [1]. GSMA has published such a coupon framework [4] enabling smartphones with Near-Field-Communication (NFC) capabilities for coupon storage and redemption, thereby excluding paper-based coupons and phones without NFC antennas.

In order to move coupon interoperability forward, technical alignment and experience sharing from real-world implementations is necessary, which is the primary contribution of this paper, as it extends [3] by providing actual API definitions from a practical field implementation. Thus, the non-profit standard body GS1 suggests a concept [5] based on the Global Coupon Number (GCN) standard [1], which supports digital and paper-based coupons [44]. The GS1 GCN concept has been subject to a single-retailer based pilot implementation [2] that does not yet allow for interoperability of coupon distribution and validation between multiple retailers and brands. It furthermore lacks an actual definition of standardized distribution and validation APIs [2], required to share relevant coupon information in a decentralized, crossorganizational setup. Moreover, GS1 published the conceptual idea to extend the Digital Coupon Management Framework (DCMF) [5] through API definitions enabling interoperability of coupons [3]. Said framework however has also not yet been publicly specified on a technical implementation level. Digital Coupon Management Framework

Before introducing the proposed system architecture and API definitions (i.e. Coupon Distribution API, Coupon Validation API, Event Repository API, Campaign Management API), we define the involved stakeholders of the corresponding, underlying DCMF architecture [5], as illustrated in figure 1.

- Coupon Distributor (one or more): A physical service or digital application service that distributes coupons to end consumers. Typically, Internet Application Providers (i.e.

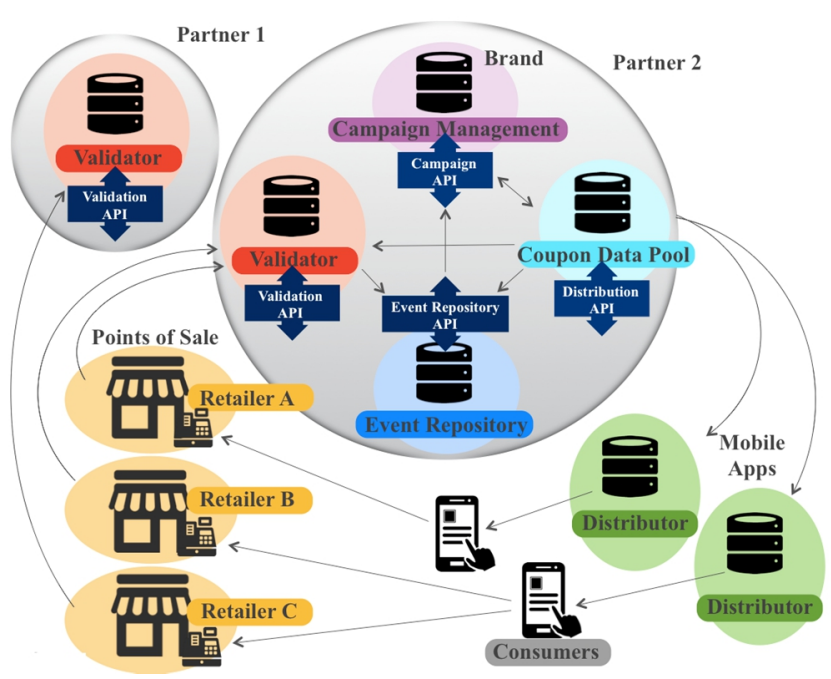

Figure 1: Proposed Interfaces for Coupon Interoperability

mobile applications) or printed magazines distribute coupons. This is the role of Offer Distributor in the DCMF. Distributers receive available coupons via querying the

'Coupon Distribution API' from Coupon Data Pools and can send coupons to end customers, e.g. via push notification, wallet inclusion, beacon triggers, or (serialized) paper coupons. Coupon distributers create the front-end to consumers and the visual/technical representation of the coupon.

- Retailer/Point-of-Sale (POS) Integrator/Operator (one or more) (in the DMCF referred to as Offer Awarder [5]): A retail integrator is either integrated into the POS System of the Retailer or the Retailer itself. End consumers present their paper- or app-based coupons to the POS, where the coupon identifier is registered. The integrator therefore hosts a service that receives coupons at the POS and checks the validity of the coupons via the 'Coupon Validation API'.

- Validator (one or more) (in the DMCF referred to as Offer Validator [5]): A service adhering to the 'Coupon Validation API' standard, receiving requests from POS Integrators and sending replies regarding the validity of a coupon back. A validator manages the validation of a coupon, and relies on information from the Coupon Data Pool (CDP), Campaign Management as well as Event repository to verify the authenticity of a coupon that was handed over through the 'Coupon Validation API'.

- Coupon Data Pool (one or more) (CDP): A CDP is a centralized coupon management platform with the primary function of coupon data integration and synchronization in the (digital) coupon value chain. It may also provide a combination of additional services such as coupon issuance (including serialization), campaign management, coupon distribution, coupon validation and coupon tracking, automated clearing of coupons and reporting services.

- Campaign Management (one or more): Brands usually have Campaign Management tools, which can launch and manage coupon campaigns. Campaign Managers can leverage logs from previous campaigns gathered from the 
Event Repository (ER) and create new coupons that are logged in their Coupon Data Pool.

- Event Repository (one or more): An event repository is a database to capture and share information about the movement and statuses of coupon objects, e.g. events that result from queries to the 'Coupon Validation API'. The events that are registered can include valid/failed redemptions per campaign, different reasons for failures.

If multiple representations of stakeholder types exist simultaneously within a coupon ecosystem, Domain Name Systems (DNS) [45] - similar to accessing websites on the internet - can be requested to identify the respective server from which to access the relevant information (e.g. matching the correct Event Repository to a coupon identifier).

\section{Proposed INTEROPERABLE SYSTEM ARCHITECTURE}

In [3], the following APIs are seen as necessary for an interoperable coupon ecosystem, but remain so far ill-defined in the literature [1]-[4]. We thus elaborate on these elements in more detail and propose a re-usable design of the 'Coupon Validation API' in the next chapter, as it enables the coupon interoperability (Fig. 1).

\section{A. Coupon Distribution API}

For Coupon Distributors (e.g. mobile applications, print magazines, etc.) to access available coupon campaigns from Coupon Data Pools (CDP) (i.e. retailers, brands, etc.) (Fig. 1), a standardized interface shall allow constant, up-to-date, realtime access to currently available campaign data. Because of growing smartphone adoption and application of omnichannel strategies, the number of relationships between CDPs and relevant Coupon Distributor within national and international coupon ecosystems is already large and expected to grow substantially [3]. Therefore, standardization of coupon distribution can lead to efficiency, as relevant information can be accessed easier, quicker and by lower cost due to reduced implementation costs, in order to set up new partnerships between apps, brands and retailers. The 'Coupon Distribution API' shall provide requesting parties the following information: First, the reply contains necessary information per coupon object [1], [5], e.g. offer details, coupon artwork, terms and conditions, participating store locations, required information for validation and identification. Further, the respective Coupon Distributor shall be revealed via identifier in the API request to include distributor-specific information in the reply, such as how many coupons shall be made available for the respective campaign, and rules on how to distribute and serialize these campaign's coupons to the Distributor's audience (i.e. number of coupons per user, participating regions, limits on number of validations, etc.). Besides distributing campaign information to coupon distributors, the Coupon Distribution API also updates the ecosystem's Event Repository on outstanding coupons, enabling the calculation of campaign redemption rates and validation of newly created coupons.

\section{B. Campaign API}

Coupon sponsors (e.g. brands or retailers), usually plan their coupon campaigns with dedicated tools, i.e. campaign management systems [3] (Fig. 1). In order to make relevant coupon information of current and upcoming campaigns automatically available to the Coupon Distributors, they must share their campaign information with Coupon Data Pools via the 'Campaign API' (Fig. 1). It shares relevant campaign data, i.e. offer details, coupon objects [1], [5], distribution details, i.e. who shall distribute which quantities in which duration and region, such that the 'Coupon Distribution API' can allocate relevant coupon objects to respective Coupon Distributors. Further, the Campaign API receives information from the Event Repository on redemption and distribution statistics for relevant campaigns of the respective coupon sponsor (Fig. 1).

\section{Event Repository API}

The Event Repositories contain event logs of the various lifecycle stages per coupon, i.e. from creation, via distribution to valid or invalid redemption (Fig. 1). The corresponding 'Event Repository API' of each Event Repository allows searching for (entering of) such coupon-specific events (i.e. timestamp, time zone, coupon identifier, event type, etc.) by Validators (Distributors). Further, sharing information on distribution and redemption statistics per campaign allows to compute marketing-relevant performance indicators for campaign management and clearance of redeemed coupons.

\section{Coupon Validation API}

In order to validate distributed coupons from consumers at the POS, the 'Coupon Validation API' as interface through which a POS Integrator (Retailer) (Fig. 1) queries the validity status of a consumer's (serialized) coupon, is required. Therefore, after scanning the coupon at the till, the POS contacts the 'Coupon Validation API' in order to inquire the validity status of the coupon. For optimization of validation response time in a fragmented coupon ecosystem during the time-sensitive checkout process, a DNS-like registry can redirect such requests to the correct Validator of the respective coupon. The API service receives requests from POS integrators and sends replies regarding the validity of a coupon directly back to the POS: These replies can yield valid/invalid (e.g. expired, product not in basket, etc.) and also fulfill operations such as redeem/unredeem/check validity. After a coupon is validated or rejected by the Coupon Validation API, the transaction's events are recorded in the Event Repository.

\section{E. Further APIs}

Besides the APIs introduced above, further interfaces are included in current concepts of future coupon ecosystems [3]. Nevertheless, they are not as frequently used between disjunctive stakeholders, therefore less relevant for interoperability and excluded in this paper.

\section{IMPLEMENTED INTEROPERABLE COUPON VALIDATION}

In the context of this study, a proof-of-concept implementation was conducted to prove the feasibility of applying standardized 'Coupon Validation API' across different retailers for coupon validation of a coupon within a real-world, 
cross-retailer environment. Therefore, a practical pilot implementation was set up in the city of Birmingham from September to December 2015, involving existing stakeholders of the couponing industry in the United Kingdom (Table 1). The proof-of-concept's coupon campaign was sponsored by Coca Cola and designed as a mobile-mediated coupon, using GS1's GCN coupon identifying schema [1] (Fig. 2). In the pilot, the coupon was valid for a free can of Coca-Cola along with a purchase at participating stores. The coupons were registered at the POS System in the Coupon Data Pool (CDP) prior to distribution.

\begin{tabular}{|c|c|c|}
\hline \multirow{2}{*}{ Stakeholder } & \multicolumn{2}{|r|}{ Involvement } \\
\hline & Role & Contribution \\
\hline Coca Cola & Brand & Sponsoring Coupon \\
\hline PARTNER1 & Validator & Providing Coupon Validation API 1 \\
\hline PARTNER2 & Validator & $\begin{array}{l}\text { Providing Coupon Validation API } 2 \\
\text { Providing Coupon Data Pool } \\
\text { Providing Campaign Management } \\
\text { Providing Event Repository }\end{array}$ \\
\hline APP1 ... APP8 & $\begin{array}{l}\text { Distributors } \\
\text { (total: } 8 \\
\text { apps) }\end{array}$ & Distributing Coupons to Consumers \\
\hline $\begin{array}{l}\text { RETAILER1 ... } \\
\text { RETAILER17 }\end{array}$ & $\begin{array}{l}\text { Retailers } \\
\text { (total: } 17 \text { in- } \\
\text { dependent } \\
\text { retailer } \\
\text { locations) }\end{array}$ & $\begin{array}{l}\text { Integrating Coupon Validation } \\
\text { APIs to request coupon validity } \\
\text { upon scanning coupon at POS, } \\
\text { receiving and processing coupon } \\
\text { validity status }\end{array}$ \\
\hline End users & Consumers & $\begin{array}{l}\text { Receiving coupons on mobile } \\
\text { devices, redeeming them at } \\
\text { participating retailers }\end{array}$ \\
\hline
\end{tabular}

Table 1. Participating stakeholders in pilot implementation

PARTNER2 provided the Coupon Data Pool (CDP) storing the available coupon campaign and distribution rules. The CDP allowed the eight different mobile applications to receive allocated coupon quantities for distribution towards consumers. The pilot's coupon integration included popular consumer applications in the areas of social media and couponing, and distributed coupons through geo-based campaigns via Bluetooth beacons and GPS geo-fencing. These conditions ensured that primarily users in Birmingham were targeted by the pilot's campaign.

Although the designs of participating mobile applications were heterogenic, the respective coupon presentation would adhere to design templates defined by the CDP (Fig. 2). Also, the redemption process at the 17 retailer locations was coherent, i.e. optical scan of a serialized GCN [1]. The optical scan required some of the retail locations to upgrade their optical handheld scanning device, as GCNs can be longer (i.e. up to 25 digits) than their current optical setups scanning capability, often required to scan GTINs (i.e. up to 13 digits) [38]. Upon presentation of the mobile coupon at the POS, the retailers' till would register the GCN barcode on the coupon and

\section{Figure 2: Proof-of-concept's coupon provided by Coca Cola}

automatically initiate a real-time request to the respective 'Coupon Validation API'. The validation requests compare the

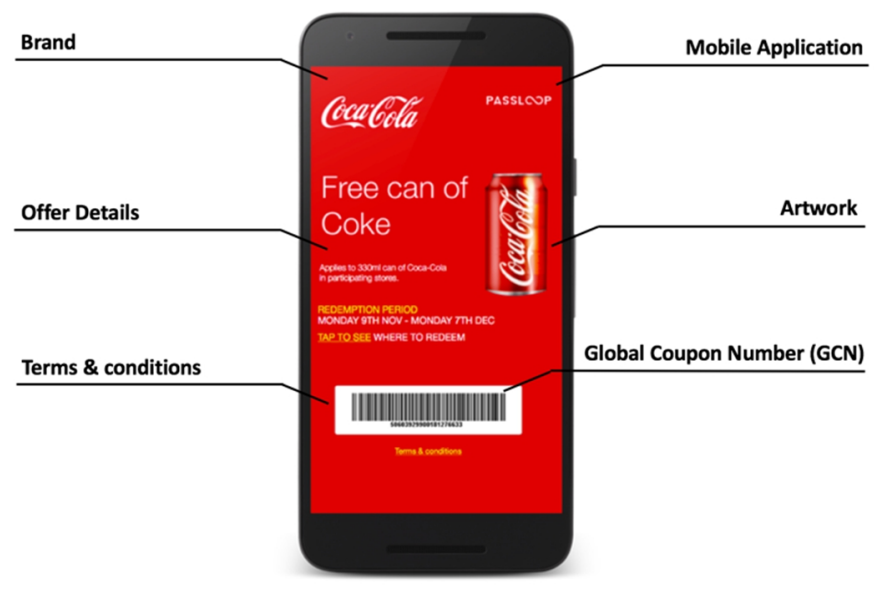

consumers' coupon against the cross-organizational, electronic event repositories. Thereby allowing POS Operators to check the validity of the presented coupon. For this process, the Digital Coupon Management Framework [5] foresees two typical scenarios involving a loyalty system and an offer database. For the sake of simplicity, the 'Coupon Validation API' of this proof-of-concept however only accounted for the latter. It is important to note that two conditions were assumed in the context of this pilot study. For one, the POS integrator and the Validator have a pre-existing trust relationship for validation operations, such that they can mutually authenticate one another and the POS can query the Validator (i.e. has access to the 'Coupon Validation API' via a valid API key). Second, the Validator has pre-existing trust relationships with the CDP and Event Repository and is thus authorized to query coupon lifecycle information.

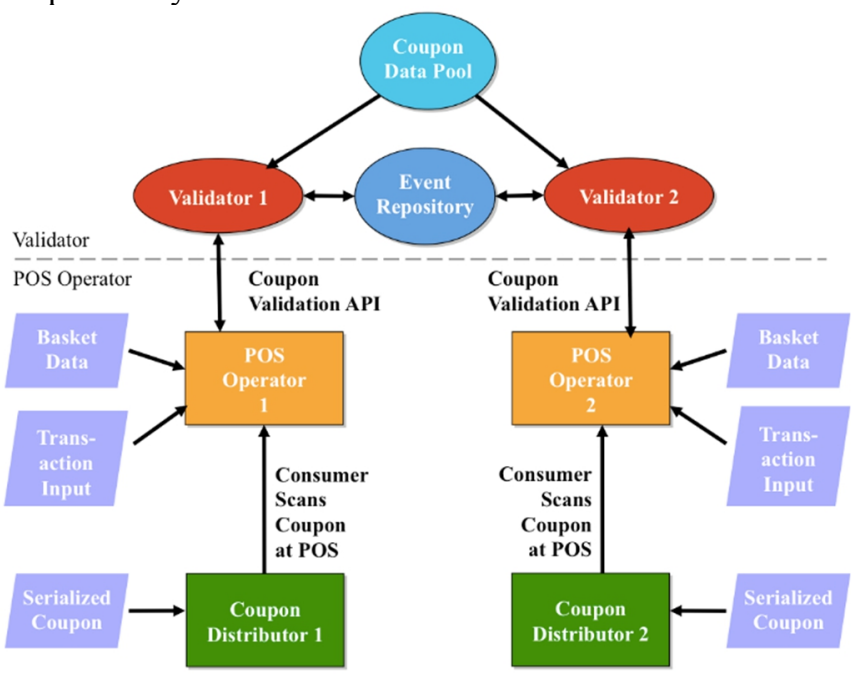

Figure 3: Information Flow in Coupon Validation API

The overall purpose of the 'Coupon Validation API' service is to make the necessary backend interactions with the Coupon Data Pool and Event Repository to get coupon data, compute requested operations and return the information requested by the POS client (Fig. 3). The 'Coupon Validation API' therefore queries the CDP in real-time to verify, redeem or unredeem corresponding coupons based on its relative data, 
such as transaction identifier, current timestamp, store location identifier, coupon identifier, products in basket (optional basket verification).

To enable interoperability, such that multiple POS integrators at separate retailers to use the same 'Coupon Validation API', the following Validation methods were implemented in the proof-of-concept's architecture as RESTful APIs. The respective requests and responses were delivered JSON payloads (Fig. 4):

\section{A. Coupon Verification Method}

During the checkout process, the POS Operator requests the Validation Service to verify whether the coupon provided is valid. Therefore, the coupon status is given, without activating coupon redemption. Sequentially, the POS Operator issues a coupon verification query to the 'Coupon Validator API', which includes a transaction identifier, list of coupons that were scanned and need to be verified, merchant and store identifiers, local as well as a server timestamp of the POS Operator in case of a potentially incorrect local timestamp at the till. In addition, an optional product basket that was scanned and contains potentially discountable products can be added to the Coupon Verification Request, allowing for basket-based coupon verification. The 'Coupon Validation API' reply in turn computes and provides the verification status of the coupon to the POS Operator, including the respective transaction identifier, the array of coupons involved, a result code and message for each coupon (e.g. valid, expired, lost/stolen, timing invalid, promotion ended, validation count exceeded, store invalid, product not in basket, cannot-unredeem, etc.), server timestamp and offer details (e.g. discount, product identifier, etc.). The POS Operator subsequently processes the obtained result, e.g. present the status to the consumer or redeem the coupon in a separate step (i.e. trigger the coupon redeem method).

\section{B. Coupon Redeem Method}

To redeem a presented coupon, the POS Operator requests the Validator via the 'Coupon Validation API' who first validates and then redeems the respective coupon, if valid. In this sense, the request and reply function are defined similarly to the Coupon Verification Method. Unlike in the former approach, the Validator however redeems the coupon by redeeming it within the Event Repository. Necessarily, the result code 'redeemed' is then added to the reply. This step in turn enables the POS Operator to process the results, i.e. deduct the prices and recalculate value added taxes according to the offer details. In case multiple redemptions alternatives are possible (e.g. $50 \%$ price reduction off a single item, with multiple items in the basket), the Coupon Redeem Method calculates the optimal redemption that saves the consumer the most money. In case of unsuccessful redemption, the corresponding error message is sent back to the POS Operator (e.g. offer expired, item not in basket, store not participating). If the Coupon Validation API service signals validity, the consumer would be granted the benefits of the coupon automatically via the POS Operating Service.

\section{Coupon Unredeem Method}

For unintended redemptions, the unredeem method can undo previously conducted redemption of a scanned coupons. In such a case, the POS operator sends transaction identifier, list of coupons to be unredeemed, merchant and store identifier, timestamp of POS, server timestamp, original transaction identifier to unredeem to the 'Coupon Validation API'. The corresponding reply contains the transaction identifier, the array of coupons, result codes and messages, offer details and server time stamp. Upon successful unredemption and settlement of the price difference, the corresponding Coupon Data Pool and Event Repository need to be updated by the 'Coupon Validation API', such that the respective coupon is valid again in the future.

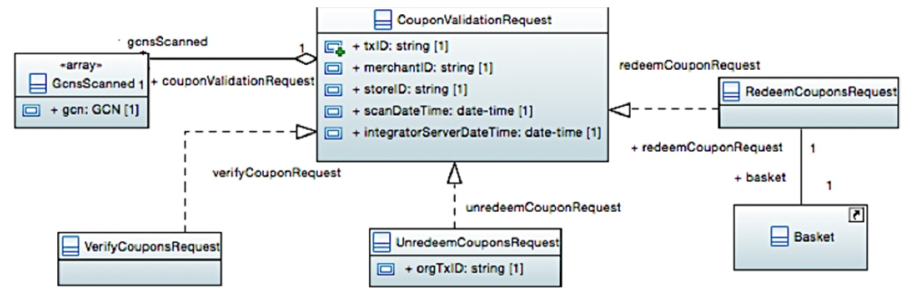

Figure 4. Overview of Coupon Validation Methods: Coupon Verification, Coupon Redemption, Coupon Unredemption

\section{RESULTS}

Unfortunately, the pilot consortium decided not to publish concrete adoption and redemption numbers. Nonetheless, results of the proof-of-concept pilot in Birmingham are still manifold and interesting to discuss.

First, the pilot proves feasibility of a real-time, interoperable, cross-retailer 'Coupon Validation API' within a real-world environment. The 17 POS outlets belong to 16 small independent owners and one multinational supermarket chain, and therefore represent an ideal environment for an interoperable coupon ecosystem. The two POS integrators that manage the till systems for the 17 retail locations found it rather straightforward to build and connect to the 'Coupon Validation API' and to accept the mobile-distributed coupons from consumers that were brought to their stores through thirdparty mobile applications.

Second, the pilot has shown that interoperability for coupons is very valuable for the mobile application community. Often, mobile applications struggle to find a commercially viable long-term business model, despite owning large user audiences and knowing the exact location and consumption preferences of a user. Implementing digital coupons would allow application providers to participate in the retail ecosystem and earn revenue without charging end users a usage fee, a promising strategy for mobile applications. The eight mobile application providers participating in this study mentioned that the main reason to participate in the interoperability pilot was their aim to monetize their audience through coupon distribution in the future.

Third, consumers benefit from interoperability through increased convenience as the average distance to a relevant 
retail outlet is reduced: Since the pilot's interoperability allowed consumers to redeem the coupon across multiple retailer outlets within Birmingham, it was more convenient for users to find a close-by store, rather than having to bridge the distance to one specific store, which might be located far away.

Fourth, applied design science approach helped to construct functional cross-organizational interfaces: as information exchange between stakeholders along a coupon value chain is often critical for both strategic and political reasons, the solution lies in consequent, iterative collaboration between experts of different fields and organizations, such as retailers, mobile consumer apps, POS Operators and consumers, in order to construct the interfaces such that all stakeholders agree to.

Lastly, the learnings made in the course of the pilot show that while brands are supportive of a cross-retailer coupon ecosystem, some retailers are hesitant to lose influence and fear that their impact on the retail value chain is weakened by an open, standardized couponing standard. As the pressure for market shares in the UK is tightening, eventually the pressure to innovate could lead retailers into accepting interoperable, cross-retailer coupons.

\section{DISCUSSION}

The study's main objective to test the technical feasibility of integrating the conceptually introduced cross-organizational information interfaces [2], [3] within a real-world environment, was fully achieved through implementation of this pilot. The qualitative results indicate that interoperability brings value to coupon distributors (i.e. more distribution measures and less effort to set up new partnerships with apps and retailers), brands (i.e. more transparent redemption analytics per app or store), consumers (i.e. convenience, as coupons are now redeemable at more store outlets) and to some extent even retailers (e.g. mobile application users being drawn to their stores). Further, the proof-of-concept has proven that real-time APIs are stable and can be used reliably across retailers and brands in order to enable a cross-retailer couponing landscape, as there was no single network interruption during the pilot. However, for full interoperability, the retail landscape needs to upgrade their technological infrastructure, e.g. optical scanners and technical application interfaces, which can be seen as onetime investments for retailers. Further, the adoption of established retail standards, architectural frameworks and system principles, such as GTIN, GCN, GLN [2], [3], seem critical in establishing an interoperable, cross-retailer couponing ecosystem, as the many already existing, proprietary approaches have so far failed to be implemented in a real-world scenario. Further learnings include that consumers expect digital coupons to be integrated into mobile wallet apps, and that brands and retailers are supportive in providing the test field for digital coupons. Further, it can be foreseen that mobile application providers, such as Google or Facebook are very interested in integrating coupons into their consumer-facing applications in order to extend their business model into retail.

Limitations of this work include the fact that more quantitative measures are required to assess the full potential of interoperability in the couponing landscape in more detail. As the coupon distribution is conducted by third-party applications and coupon redemption statistics are of sensitive nature for brands and retailers and therefore not to be published in detail in this pilot, further, more rigor and more quantitative experiments and results are required and intended to be published in the future. Besides quantitative assessments of validation response times and redemption rate comparisons between (non-)interoperable coupons, necessary future work also includes conceptualizing interfaces for validating coupons based on loyalty card identifiers or other identifiers. In addition, information interfaces for non-trusted relationships or network hubs need to be addressed, as interoperability in the ideal case would allow consumers to redeem coupons at any retail outlet. Last but not least, further information exchange APIs besides Coupon Validation (Fig. 1) are intended to be defined, implemented and tested in the same retail environment in Birmingham in the future.

\section{CONCLUSION}

This proof-of-concept of coupon interoperability contributes to theory and practice through extending the current, theoretic concepts of information standardization [1] and exchange [3], [5] in the coupon ecosystem through a first, crossorganizational implementation of standardized APIs. The preliminary results prove that enabling interoperability of digital coupons is feasible for different stakeholders, from small one-shop-kiosks to large multinational brand through the support of POS Operators. Additionally, interoperability of coupons seems to potentially become a key property of digital (and paper-based) coupons in the future, as customers want them to be redeemable independent of the retail outlet and chain. Further, the results prove that technical standards for cross-organizational information exchange are critical for interoperability in the couponing ecosystem. Therefore, (global) industry alignment in the very fragmented and for the most part not-standardized couponing ecosystem is needed on the further design and prioritization on standards development to achieve global interoperability. Especially as digital couponing is growing around the globe [21], [28], standard bodies such as GS1 should play a leading role in the standardization of cross-organizational coupon information exchange, which would require key stakeholder consolidation at the global level and continued work on standards, such as the DCMF [5] and corresponding API standards, in order to enable retailers and brands to distribute and validate digital coupons in real-time.

The objective of the pilot was primarily to prove feasibility of interoperable coupon framework. With this foundation now in place, future work on subsequent pilots will include building and testing further cross-organizational couponing information exchange interfaces and acceptance studies through further large-scale campaigns with multiple brands and retailers. 


\section{REFERENCES}

[1] GS1, "Global Coupon Number (GCN): Specification and Definition," 2015. [Online]. Available: http://www.gs1.org/global-coupon-numbergcn. [Accessed: 18-Jun-2016].

[2] J. Araújo, G. P. Dias, H. Gomes, J. Gonçalves, D. Magueta, F. Marques, C. Martins, and M. Rodrigues, "An Integrated Service Solution for Digital Discount Coupons Processing," Adv. Intell. Syst. Comput., vol. 353, no. April, pp. III-IV, 2015.

[3] GSMA and GS1, "Digital Commerce in Retail: Mass Distribution and Acceptance of Mobile Couponing," 2015. [Online]. Available: https://www.gs1uk.org/ /media/documents/marketing-

documents/gs198325mobile_coupon_brochure_0215.pdf?la $=$ en. [Accessed: 18-Jun-2016].

[4] GSMA, "Mobile Commerce, NFC Coupons and Loyalty Acceptance Technical Proposal," 2014. [Online]. Available: http://www.gsma.com/digitalcommerce/nfc-15-mobile-commerce-nfccoupons-and-loyalty-acceptance-technical-proposal. [Accessed: 18-Jun2016].

[5] GS1, "Digital Coupon Management Standard Specification," 2012. [Online]. Available: http://www.gs1.org/digital-coupons/digital-couponstandard/1. [Accessed: 18-Jun-2016].

[6] J. Bughin, M. Chui, and J. Manyika, "An Executive's Guide to the Internet of Things," McKinsey Glob. Inst., no. March 2010, p. 9, 2015.

[7] G. Aloi, G. Caliciuri, G. Fortino, R. Gravina, P. Pace, W. Russo, and C. Savaglio, "A Mobile Multi-Technology Gateway to Enable IoT Interoperability," 2016 IEEE First Int. Conf. Internet-of-Things Des. Implement., pp. 259-264, 2016.

[8] I. Ganchev and M. O'Droma, "A Generic IoT Architecture for Smart Cities," 25th IET Irish Signals Syst. Conf. 20142014 China-irel. Int. Conf. Inf. Communities Technol. (ISSC 2014/CIICT 2014), pp. 196199, 2014.

[9] F. K. Santoso and N. C. H. Vun, "Securing IoT for smart home system," in Proceedings of the International Symposium on Consumer Electronics, ISCE, 2015, vol. 2015-Augus.

[10] S. Kim, J. Y. Hong, S. Kim, S. H. Kim, J. H. Kim, and J. Chun, "Restful Design and Implementation of Smart Appliances for Smart Home," in Proceedings - 2014 IEEE International Conference on Ubiquitous Intelligence and Computing, 2015, pp. 717-722.

[11] M. Blackstock and R. Lea, "IoT Interoperability: A Hub-based Approach," 2014 Int. Conf. Internet Things, IOT 2014, pp. 79-84, 2014.

[12] M. Díaz, C. Martín, and B. Rubio, "State-of-the-art, challenges, and open issues in the integration of Internet of things and cloud computing," J. Netw. Comput. Appl., vol. 67, pp. 99-117, 2015.

[13] M. Su, X. Zheng, and L. Sun, "Coupon Trading and its Impacts on Consumer Purchase and Firm Profits," J. Retail., vol. 90, no. 1, pp. 4061, 2014.

[14] Intelligent-Clearing-Network, "Validation and Electronic Clearing of Paper Coupons at POS Solves the Problems of Mis/Malredemption and Fraud for the Coupon Industry." .

[15] A. Dickinger and M. Kleijnen, "Coupons going wireless: Determinants of consumer intentions to redeem mobile coupons," J. Interact. Mark., vol. 22, no. 3, pp. 23-39, 2008.

[16] X. Wang, J.-H. Zhang, and X.-G. Wu, "Determinants of Tourism Coupon Redemption," J. Travel Tour. Mark., vol. 8408, no. November, pp. 1-13, 2014.

[17] A. C. Nielsen Jr., “The Impact of Retail Coupons,” J. Mark., vol. 29, no. 4, pp. 11-15, 1965.

[18] L. L. Price, L. F. Feick, and A. Guskey-Federouch, "Couponing Behaviors of the Market Maven: Profile of a Super Couponer.," Adv. Consum. Res., vol. 15, no. 1, pp. 354-359, 1988

[19] Accessdevelopment.com, "Coupon Statistics: The Ultimate Collection," 2016. [Online]. Available: http://blog.accessdevelopment.com/ultimatecollection-coupon-statistics. [Accessed: 18-Jun-2016].

[20] INMAR, "Improving Economy, Increasing Shopper Demand for Digital Offers Impacting Coupon Use," 2016. [Online]. Available: http://www.inmar.com/press-release/improving-economy-increasingshopper-demand-for-digital-offers-impacting-coupon-use/.
[21] Statista, "Facts on coupon market trends in the United States," 2016. [Online]. Available: http://www.statista.com/topics/1156/couponmarket-trends-in-the-united-states/. [Accessed: 18-Jun-2016].

[22] K. Jung, B. Y. Lee, and M. G. Korea, "Online VS . Offline Coupon Redemption Behaviors,” Int. Bus., vol. 9, no. 12, pp. 23-37, 2010.

[23] K. Bawa, S. S. Srinivasan, and R. K. Srivastava, "Coupon Attractiveness and Coupon Proneness: A Framework for Modeling Redemption," J. Mark. Res., vol. 34, pp. 517-525, 1997.

[24] H. A. Roehm and M. L. Roehm, "The relationship between FSI advertising style and coupon redemption," Mark. Lett., vol. 18, no. 4, pp. 237-247, 2007.

[25] A. Musalem, E. T. Bradlow, and J. S. Raju, "Who's Got the Coupon? Estimating Consumer Preferences and Coupon Usage from Aggregate Information,” J. Mark. Res., vol. 45, no. December, pp. 715-730, 2008.

[26] T. Mutanen, S. Nousiainen, and H. Liang, "Personalized Recommendation on Discount Coupons.," Annu. Int. Conf. Comput. Sci. Educ. Innov. Technol., pp. 127-132, 2010.

[27] V. Ramaswamy, S. S. Srinivasan, and S. Srini, "Coupon Characteristics and Redemption Intentions: A Segment-Level Analysis.," Psychol. Mark., vol. 15, no. January 1998, pp. 59-80, 1998.

[28] V. Young, "Digital Coupons Influencing Purchase Behavior,” Women's Wear Daily, 2014

[29] V. Shankar and S. Balasubramanian, "Mobile Marketing: A Synthesis and Prognosis," J. Interact. Mark., vol. 23, no. 2, pp. 118-129, May 2009.

[30] H. K. Cheng and K. Dogan, "Customer-Centric Marketing with Internet Coupons,” Decis. Support Syst., vol. 44, pp. 606-620, 2008.

[31] A. Muk, "Perceptions of mobile coupons: A cross-national study," J Direct, Data Digit. Mark. Pract., vol. 13, no. 4, pp. 311-324, 2012.

[32] D. Cameron, C. Gregory, and D. Battaglia, "Nielsen personalizes the mobile shopping app: If you build the technology, they will come," J. Advert. Res., vol. 52, no. 3, 2012.

[33] Criteo, "State of Mobile Commerce," no. October, p. 41, 2015.

[34] Statista, "Number of mobile coupon users in the United States from 2012 to 2016 (in millions)." [Online]. Available: http://www.statista.com/statistics/275670/adult-mobile-coupon-users-inthe-united-states/. [Accessed: 13-Jun-2016].

[35] S. Jayasingh and U. Eze, “An empirical analysis of consumer behavioral intention toward mobile coupons in Malaysia,” Int. J. Bus. ..., vol. 4, no. 2, pp. 221-242, 2009

[36] D. Chaffey, "Mobile Marketing Statistics 2016," Smart Insights, 2015 [Online]. Available: http:/www.smartinsights.com/mobilemarketing/mobile-marketing-analytics/mobile-marketing-statistics/.

[37] C. Blundo, S. Cimato, and A. De Bonis, "Secure e-coupons," Electron. Commer. Res., vol. 5, no. 1, pp. 117-139, 2005.

[38] D. L. Brock, "White Paper: Integrating the Electronic Product Code (EPC) and the Global Trade Item Number (GTIN)," MIT Auto-ID Cent., no. February 1, pp. 1-25, 2001.

[39] Coupons.com, "Patent Application: Digital Coupon Clearinghouse," Patent publication number: US20120284107 A1, 2012.

[40] Amazon, "Digital Coupon System," Patent publication number: US20120136712 A1, 2012.

[41] T. Robards, "Selective Communication for Digital Coupon Distribution," Patent publication number: US20120289255 A1, 2012.

[42] Apple, "Integrated Coupon Storage, Discovery, and Redemption System," Patent publication number: US20120323664 A1.

[43] D. R. Hammerstad and K. J. Kaaz, "Variable Value Coupons," Patent publication number US20120310719 A1, 2012.

[44] GS1, "GS1 Position Paper on Barcodes for Mobile Applications," 2012. [Online]. Available: http://www.gs1.org/docs/gs1source/GS1_Mobile_Barcodes_Position_Paper.pdf. [Accessed: 18-Jun2016].

[45] S. Josefsson, "Domain Name System Uniform Resource Identifiers," RFC, 2006. 